\title{
No selection for cheating in a natural meta- population of rhizobia
}

\author{
Megan Frederickson
}

Version Post-print/accepted manuscript

Citation Frederickson ME. No selection for cheating in a natural meta-population of (published rhizobia. Ecol Lett. 2020 Feb;23(2):409-411. doi: 10.1111/ele.13293. PMID: version) 31898406.

Publisher's This is the peer reviewed version of the following article:

Statement No selection for cheating in a natural meta-population of rhizobia, which has been published in final form at

https://doi.org/10.1111/ele.13293 This article may be used for noncommercial purposes in accordance with Wiley Terms and Conditions for Use of Self-Archived Versions.

\section{How to cite TSpace items}

Always cite the published version, so the author(s) will receive recognition through services that track citation counts, e.g. Scopus. If you need to cite the page number of the author manuscript from TSpace because you cannot access the published version, then cite the TSpace version in addition to the published version using the permanent URI (handle) found on the record page.

This article was made openly accessible by $U$ of $T$ Faculty. Please tell us how this access benefits you. Your story matters. 


\title{
No selection for cheating in a natural meta-population of rhizobia
}

\author{
Megan E. Frederickson ${ }^{1 *}$
}

${ }^{1}$ Department of Ecology and Evolutionary Biology, University of Toronto, Toronto, ON, M5S 3B2, Canada

* Author for correspondence: m.frederickson@utoronto.ca

Running title: No selection for cheating

Length: 783 words in main text, 48 words in abstract, and 2 figures

\section{ABSTRACT}

2 Whether natural selection favors 'cheating' in mutualisms is hotly debated. Gano-

3 Cohen et al. (2019a) report a negative correlation between fitness and mutualist

4 quality in rhizobia, suggesting that rhizobia evolve to cheat. However, re-analysis

5 of their data shows that the correlation is an artifact of unequal sampling across

6 populations.

\section{MAIN TEXT}

8 Measuring fitness is tricky, and measuring the fitnesses of two interacting organisms

9 is doubly difficult. We must rise to the challenge, though, if we want to understand 10 whether partners in mutualisms are under selection to 'cheat.'

11 Selection for cheating should manifest as a negative genetic covariance between 12 an organism's fitness and its quality as a mutualist, measured as the organism's 13 effect on its partner's fitness (Friesen, 2012; Porter \& Simms, 2014; Jones et al., 
2015). Gano-Cohen et al. (2019a) conclude that rhizobia cheat based on finding a negative correlation between rhizobium fitness and symbiotic effectiveness (see their Fig. 5). They note that the pattern is largely driven by two strains, 155 and 156, that provide little benefit to hosts, but have high relative abundance in the field. However, my re-analysis of their rhizobium fitness data casts doubt on their results. They collected Acmispon strigosus plants from six natural populations and isolated Bradyrhizobium strains from their nodules. They individually tested 26 strains against several $A$. strigosus genotypes in a large greenhouse experiment. They measured plant growth to quantify each strain's symbiotic effectiveness, as well as nodule number and mass, which are common measures of rhizobium fitness (Heath \& Tiffin, 2007, 2009; Porter \& Simms, 2014; Batstone et al., 2017). They report that plant growth was not significantly correlated with nodule number or mass across strains. So there was no evidence of selection for cheating when rhizobium fitness and mutualist quality were measured in a common environment-the greenhouse.

Gano-Cohen et al. (2019a) then used sequence data from a previous study to estimate strain frequencies at the field sites where the bacteria were collected. They report the frequencies of Bradyrhizobium chromosomal (CHR) and 'symbiosis island' (SI) haplotypes within each population. They found that strain frequencies in the field were negatively correlated with symbiotic effectiveness in the greenhouse, mostly because 'ineffective' strains 155 and 156 were locally abundant. But here is where the problems emerge.

I downloaded their data (Gano-Cohen et al., 2019b) and discovered that for the population where strains 155 and 156 were sampled (YUC; Fig. 1), genotype frequencies were calculated from just two (SI frequencies) or seven (CHR frequencies) plants. Furthermore, highly unequal sampling results in differences in mean strain frequency among the six study populations. For both loci, genotype frequency is significantly negatively correlated with the number of plants sampled at a site (CHR: $\left.R^{2}=0.175, p=0.034 ; \mathrm{SI} ; R^{2}=0.207, p=0.023 ; \mathbf{F i g} . \mathbf{1 a}, \mathbf{b}\right)$. In fact, these relation- 
ships have slightly higher $R^{2}$ values than the negative correlations between genotype frequency and symbiotic effectiveness reported in the paper (CHR: $R^{2}=0.157$, SI: $\left.R^{2}=0.178\right)$. As the authors sampled more plants in a population, mean strain frequencies declined, suggesting that sampling additional plants regularly recovers novel rhizobia genotypes. This is further supported by plotting how the number of unique rhizobia genotypes increases as a function of the number of plants sampled (Fig. 1c,d); the curves do not plateau. Thus, high strain frequency is an artifact of inadequate sampling.

The authors sampled multiple nodules per plant, but their data shows nodules on the same plant are not independent samples of rhizobia in a population. Nodules on the same plant have fewer unique rhizobia genotypes than the same number of nodules chosen at random within a population (Fig. 1e,f). This suggests that plants 'choose' rhizobia genotypes in the wild, or that natural rhizobia populations are spatially structured (Akçay, 2017).

I accounted for differences in sampling effort among populations in two ways. My code is on GitHub (https://github.com/drfreder/selection-for-cheating). Briefly, first, I relativized rhizobium fitness within each population by dividing by the population mean, as is common when estimating selection gradients (e.g., Porter \& Simms, 2014). This ensures each population has the same mean fitness. Relationships between genotype frequency and symbiotic effectiveness become nonsignificant, with $R^{2}$ values near zero (CHR: $R^{2}=0.025, p=0.444$, SI: $R^{2}=$ $0.000, p=0.982$; Fig. $\mathbf{2 a}, \mathbf{b})$. Second, I re-sampled the data to equalize sampling effort, but not necessarily rhizobium fitness, across populations. One hundred times per population, I randomly chose two or four plants from each population to calculate SI and CHR genotype frequencies, respectively. These were the minimum number of plants used to calculate genotype frequencies in Gano-Cohen et al. (2019a). I then averaged genotype frequencies across iterations. This re-sampling also led to non-significant relationships between strain frequency and symbiotic ef- 

82 (Frederickson, 2017).

fectiveness (CHR: $R^{2}=0.100, p=0.116$, SI: $R^{2}=0.007, p=0.702$; Fig. 2c,d). These analyses shows that uneven sampling effort across populations gives rise to the negative associations between strain frequency and symbiotic effectiveness reported by Gano-Cohen et al. (2019a).

Mutualism researchers need to agree on how to measure selection on mutualist quality in empirical studies, or we will not progress in our understanding of mutualism evolution. I suggest measuring fitness and mutualist quality in a common environment, ideally in the same experiment, because pooling across populations or environments, or correlating fitness measures from one environment with trait values measured in another, can produce misleading results. The results of Gano-Cohen et al. (2019a) do not show that rhizobia are under selection to cheat, leaving the field with few empirical examples of fitness conflict between partners in mutualisms

\section{ACKNOWLEDGEMENTS}

84 I thank A. O'Brien and J. Stinchcombe for comments on an earlier draft. I receive 85 funding from the Natural Sciences and Engineering Research Council of Canada 86 (NSERC). 


\section{REFERENCES}

1.

Akçay, E. (2017). Population structure reduces benefits from partner choice in mutualistic symbiosis. Proceedings of the Royal Society B, 284, 20162317.

2.

Batstone, R.T., Dutton, E.M., Wang, D., Yang, M. \& Frederickson, M.E. (2017). The evolution of symbiont preference traits in the model legume Medicago truncatula. New Phytologist, 213, 1850-1861.

3.

Frederickson, M.E. (2017). Mutualisms Are Not on the Verge of Breakdown. Trends in Ecology and Evolution, 32, 727-734.

4 .

Friesen, M.L. (2012). Widespread fitness alignment in the legume-rhizobium symbiosis. New Phytologist, 194, 1096-1111.

5 .

Gano-Cohen, K., Wendlandt, C.E., Stokes, P.J., Blanton, M.A., Quides, K.W., Zomorrodian, A., Adinata, E.S. \& Sachs, J.L. (2019a). Interspecific conflict and the evolution of ineffective rhizobia. Ecology Letters, https://doi.org/10.1111/ele.13247.

6.

Gano-Cohen, K.A., Wendlandt, C., Stokes, P., Blanton, M., Quides, K., Zomorrodian, A., Adinata, E. \& Sachs, J. (2019b). Data from: Interspecific conflict and the evolution of ineffective rhizobia. Dryad Digital Repository, Available at: https://doi.org/10.5061/dryad.cr65269. 
7.

Heath, K.D. \& Tiffin, P. (2007). Context dependence in the coevolution of plant and rhizobial mutualists. Proceedings of the Royal Society B: Biological Sciences, $274,1905-1912$.

8. Heath, K.D. \& Tiffin, P. (2009). Stabilizing mechanisms in a legume-rhizobium mutualism. Evolution, 63, 652-662.

9. Jones, E.I., Afkhami, M.E., Akçay, E., Bronstein, J.L., Bshary, R., Frederickson, M.E., Heath, K.D., Hoeksema, J.D., Ness, J.H., Pankey, M.S., Porter, S.S., Sachs, J.L., Scharnagl, K. \& Friesen, M.L. (2015). Cheaters must prosper: reconciling theoretical and empirical perspectives on cheating in mutualism. Ecology Letters, $18,1270-1284$.

10 .

Oksanen, J., Blanchet, F.G., Friendly, M., Kindt, R., Legendre, P., McGlinn, D., Minchin, P.R., O’Hara, R.B., Simpson, G.L., Solymos, P., Stevens, M.H.H., Szoecs, E. \& Wagner, H. (2019). vegan: Community Ecology Package. R package version 2.5-4.

11.

Porter, S.S. \& Simms, E.L. (2014). Selection for cheating across disparate environments in the legume-rhizobium mutualism. Ecology Letters, 17, 1121-1129. 

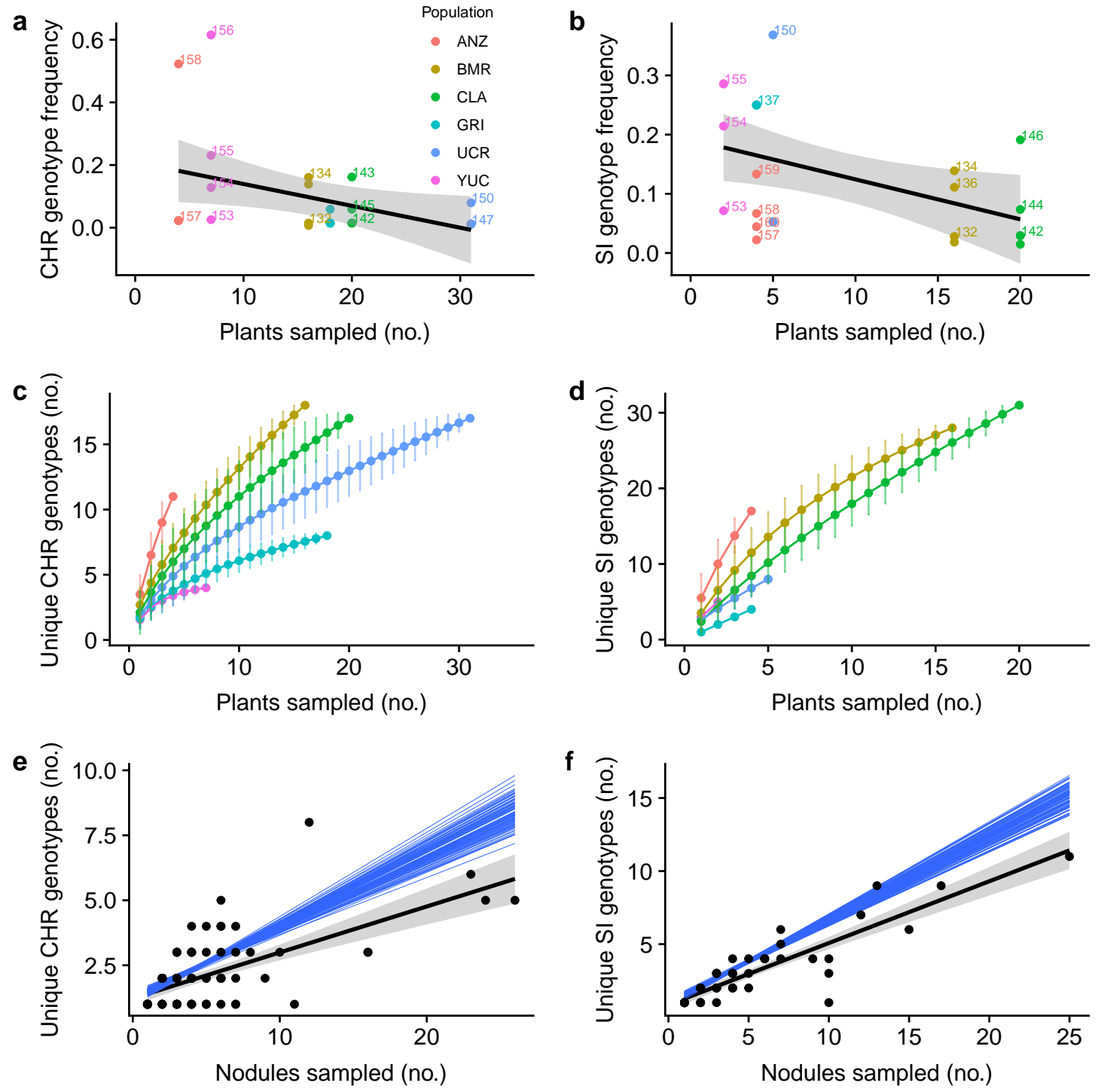

Figure 1: Top: Correlations between rhizobium CHR (a) or SI (b) genotype frequencies and sampling effort. Points are colored by population, numbers indicate strain identity, and solid lines are significant linear regressions. In $\mathbf{b}$, strain 156's SI frequency is hidden behind the dot for strain 155 because they have identical values; these strains were isolated from the same plant and share the same SI haplotype. Middle: CHR (c) or SI (c) genotype accumulation curves for the six study populations. Points and lines are colored by population (as in a), and error bars are standard deviations calculated from random permutations of the data using the specaccum function in the vegan package in R (Oksanen et al., 2019). Bottom: Number of unique CHR (e) or SI (f) genotypes in nodules from the same plant (black) or the same number of nodules sampled at random within a population (blue). Each dot is a plant, and the solid black line is the regression line fit to the original data. Each blue line is a regression line resulting from sampling the same numbers of nodules at random from each population; randomization data points not shown. Data from Gano-Cohen et al. (2019b). 

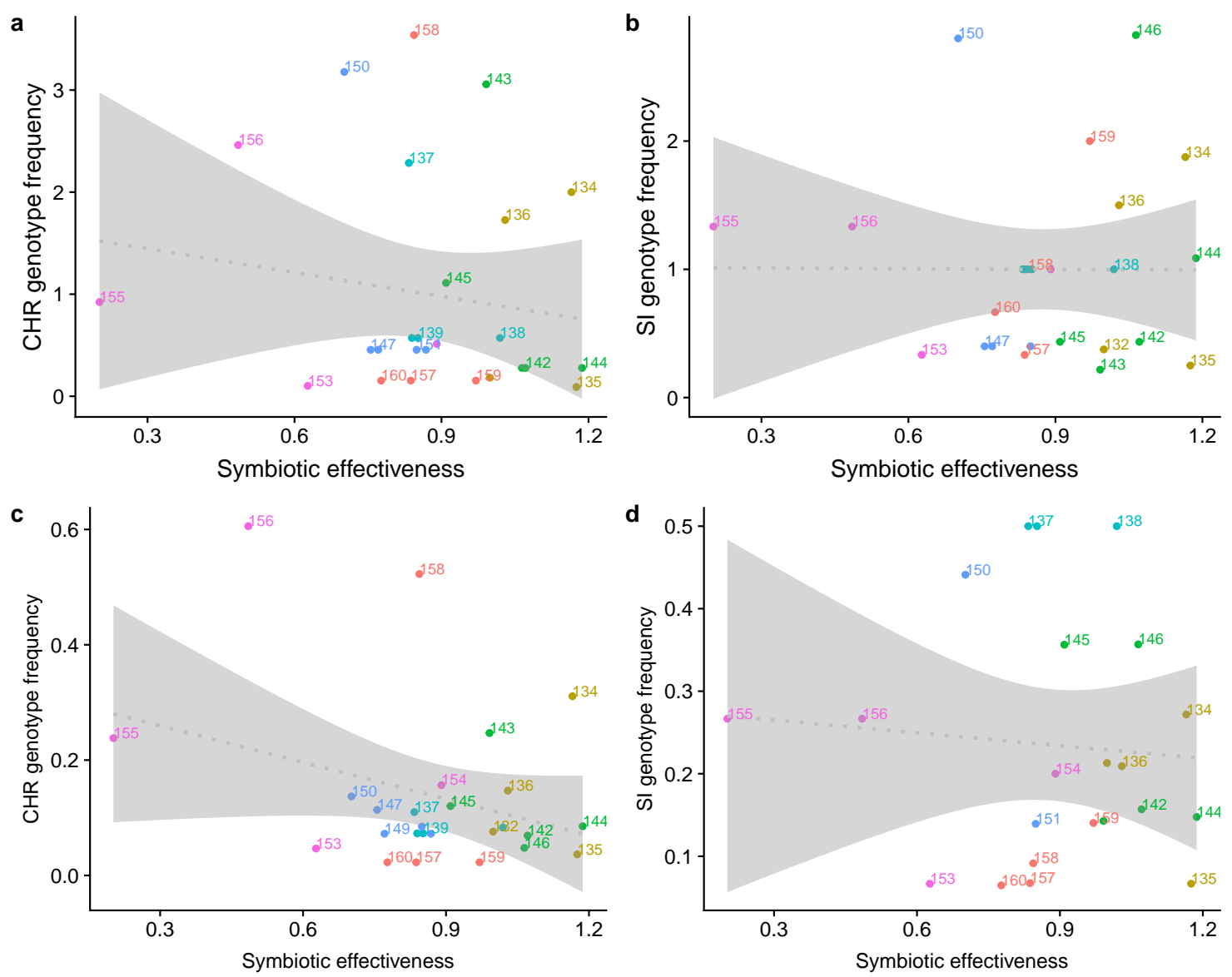

Figure 2: Top: Non-significant correlations between rhizobium CHR (a) or SI (b) genotype frequencies and symbiotic effectiveness after relativizing rhizobium fitness within populations. Bottom: Non-significant correlations between rhizobium CHR (c) or SI (d) genotype frequencies and symbiotic effectiveness after re-sampling to equalize sampling effort across populations. See text for details. In all panels, points are colored by population (as in Fig. 1a), numbers indicate strain identity, and dotted lines are non-significant linear regressions. Data from Gano-Cohen et al. (2019b). 